\title{
Approximation algorithms for the maximum Hamiltonian Path Problem with specified endpoint(s)
}

\author{
Jérôme Monnot*
}

12 September 2003

\begin{abstract}
This paper deals with the problem of constructing Hamiltonian paths of optimal weight, called $\mathrm{HPP}_{s, t}$ if the two endpoints are specified, $\mathrm{HPP}_{s}$ if only one endpoint is specified. We show that $\mathrm{HPP}_{s, t}$ is $\frac{1}{2}$-differential approximable and $\mathrm{HPP}_{s}$ is $\frac{2}{3}$-differential approximable. Moreover, we observe that these problems can not be differential approximable better than $\frac{741}{742}$.

Based upon these results, we obtain new bounds for standard ratio: a $\frac{1}{2}$-standard approximation for MAX HPP $\operatorname{HP}_{s, t}$ and a $\frac{2}{3}$ for MAX $\mathrm{HPP}_{s}$, which can be improved to $\frac{2}{3}$ for MAX $\mathrm{HPP}_{s, t}[a, 2 a]$ (all the edge weights are within an interval $[a, 2 a]$ ), to $\frac{5}{6}$ for $\operatorname{MAx}_{\operatorname{HPP}_{s}}[a, 2 a]$ and to $\frac{2}{3}$ for MiN $\mathrm{HPP}_{s, t}[a, 2 a]$, to $\frac{3}{4}$ for $\mathrm{MiN}_{\mathrm{HPP}}[a, 2 a]$.

Keywords: Approximate algorithms; Differential ratio; Complexity theory; Combinatorial optimization; Performance ratio; Analysis of Algorithms; Hamiltonian paths.
\end{abstract}

\section{Introduction}

Routing design problems are of a major importance in combinatorial optimization, and the most important ideas of algorithmic have been applied to them during the last twenty years, see Christofides [6], Fisher et al. [15], Haimovich and Rinnooy Kan [19], Kosaraju et al. [25], Hassin and Rubinstein [21] and Bazgan et al. [5]. We will be concerned with some problems closely related to the Maximum Traveling Salesman problem, namely, the problem of finding a Hamiltonian path of maximum weight. We will study two variants depending on the number of specified endpoints (one or two) of the path. MAX $\mathrm{HPP}_{s}$ and MAX HPP $\mathrm{HP}_{s, t}$ respectively denote the Hamiltonian path problem with one fixed endpoint $s \in V$ and two fixed endpoints $s, t \in V$. To our knowledge and from approximation point of view, these two latter problems have not been studied before, whereas their minimization versions have been studied by Hoogeveen [22] and Guttmann-Beck et al. [18] (in particular, it is well known that the minimization problems are NP-hard). We also deal with a variant called $\operatorname{HPP}_{s, t}[a, 2 a]$, where the edge-weights are in the set $\{a, a+1, \ldots, b-1, b\}$. Both Min- and MAx HPP $s, t$ are NPhard, even in their restricted versions with $b>a$, since they are polynomial-time Karp-reducible [24] to each other.

\footnotetext{
*monnot@lamsade.dauphine.fr, CNRS-LAMSADE,UMR 7024, Université Paris-Dauphine, France
} 
The maximum Hamiltonian path problem mainly has the same applications as the maximum traveling salesman problem since an optimum Hamiltonian path is easily converted to an optimum traveling salesman by adding one (or two) dummy vertex (vertices) with appropriate distance to all other vertices, where the specification of "appropriate" depends on the number of endpoints that have been fixed. Thus, for instance, it is known to be a relevant model for scheduling a single processor with setups arising in manufacturing, computing, VLSI design and many other applications, Lawler et al. [26]. However, this problem also has specific applications to compression data, Tarhio and Ukkonen [34] or data array clustering in DNA or marketing budgets, Hartigan [1]. For example, the maximal compression problem which arises in various compression data problems can be defined as follows: given a collection of strings $s_{1}, \ldots, s_{n}$, we seek a string $S$ such that every string in the collection is a substring of $S$ and that maximizes $\sum_{i}\left|s_{i}\right|-|S|$. In the setting, the vertices represent strings and the weight of an edge between two "strings" is set to the amount of maximum overlap between these strings. The optimal compression is equivalent to the weight of a maximum Hamiltonian path. Another application to maximum Hamiltonian path with two specified endpoints is given by the following example, Garfinkel [17]: suppose we are given a data array in the form of an $m \times n$ matrix $A=\left(a_{i, j}\right)$ that consists of elements that are either 1 if it exists a relation between row $i$ and column $j$ exists or 0 if it does not. We are interested in grouping rows and columns together in such a way that they show similar relations. For instance, consider a number of $m$ marketing techniques and $n$ products. If a marketing technique $i$ works out successfully on a product $j$, then $a_{i, j}$ gets the value 1 , and 0 otherwise. Similar marketing techniques are supposed to be successful on similar products. Therefore, clustering the techniques and the products gives insight in the relations of the marketing techniques and the products.

To formalize this, we introduce the measure of effectiveness $m e_{i, j}=a_{i, j}\left(a_{i-1, j}+a_{i+1, j}+a_{i, j-1}+\right.$ $a_{i, j+1}$ ) for each element $a_{i, j}$. To ensure their existence, we add to the matrix $A$ artificial rows of index 0 and $m+1$ and artificial columns of index 0 and $n+1$; these rows and columns contain zeroes only. The total measure of effectiveness of the matrix $A$, denoted by tme $(A)$, is computed by summing the measure of effectiveness over all elements of the matrix except for the artificial rows and columns. Thus, the goal is to find a matrix $A^{\prime}$ constructed from $A$ by permuting some rows and some columns maximizing tme $\left(A^{\prime}\right)$.

For arbitrary permutations $\rho$ and $\sigma$ of the rows and columns (representing the matrix $A^{\prime}$ ), the total measure of effectiveness of the matrix $A^{\prime}$ is tme $\left(A^{\prime}\right)=t m e_{1}\left(A^{\prime}\right)+t m e_{2}\left(A^{\prime}\right)$ where $t m e_{1}\left(A^{\prime}\right)=$ $\sum_{i=1}^{m} \sum_{j=1}^{n}\left(a_{\rho(i), \sigma(j)} \times a_{\rho(i), \sigma(j-1)}+a_{\rho(i), \sigma(j)} \times a_{\rho(i), \sigma(j+1)}\right)$ and $\operatorname{tme}_{2}\left(A^{\prime}\right)=\sum_{i=1}^{m} \sum_{j=1}^{n}\left(a_{\rho(i), \sigma(j)} \times\right.$ $\left.a_{\rho(i-1), \sigma(j)}+a_{\rho(i), \sigma(j)} \times a_{\rho(i+1), \sigma(j+1)}\right)$. Rewriting tme 1 yields: $t m e_{1}\left(A^{\prime}\right)=\sum_{j=1}^{n} \sum_{k=1}^{m} 2 a_{k, \sigma(j)} \times$ $a_{k, \sigma(j+1)}$ since on the one hand, $\rho$ is a permutation and on the other hand, $a_{k, \sigma(0)}=a_{k, \sigma(n+1)}=0$. Thus, if we define the distance $d(i, j)$ between columns $i$ and $j$ as $d(i, j)=2 \sum_{k=1}^{m} a_{k, i} \times a_{k, j}$, then we obtain the problem of finding a maximum Hamiltonian path from column 0 to column $\mathrm{n}+1$. Similarly, we can by rewriting $\mathrm{tme}_{2}\left(A^{\prime}\right)$ obtain the problem of finding a maximum Hamiltonian path from row 0 to row $\mathrm{m}+1$, where this time the distances are defined by $d(i, j)=2 \sum_{k=1}^{n} a_{i, k} \times a_{j, k}$. Finally, we see that problem of clustering a data array can be decomposed into two maximum Hamiltonian path problems, one defined on the rows and one defined on the columns. 
We focus on the design of approximation algorithms with guaranteed performance ratios, that run within polynomial time and produce sub-optimal solutions. Usually, one compares the worst case ratio (called standard ratio) of the cost of the solution generated by the algorithm to the optimal cost, in the worst-case. However, we mainly refer in this article to another ratio called differential ratio which measures the worst ratio of, on the one hand, the difference between the cost of the solution generated by the algorithm and the worst cost, and on the other hand, the difference between the optimal cost and the worst cost. This measure, studied by Aiello et al. [2], Ausiello et al. [4], Cornuejols et al. [7], Vavasis [35] (in the context of non-linear programming), Zemel [36] and more recently by Demange and Paschos [13] and Hassin and Khuller [20], leads to new algorithms taking into account the extreme solutions of the instance, and provides the opportunity to better understand these problems. There are great differences between standard and differential approximation for the maximum Hamiltonian path problems. For instance, we can easily prove that the Nearest Neighbor Heuristic (see Fisher et al. [15] or Monnot [29]) is $\frac{1}{2}$-standard approximable for MAX $\mathrm{HPP}_{s}$ and is $\frac{1}{3}$-standard approximable for MAX $\mathrm{HPP}_{s, t}$ or that we have a trivial standard approximation scheme for MAX $\operatorname{HPP}_{s, t}[n ; n+1]$ whereas, the Nearest Neighbor Heuristic is not a differential approximation with any constant ratio for $\mathrm{MAX}_{\mathrm{HPP}_{s}}$ and $\mathrm{MAX} \mathrm{HPP}_{s, t}[n ; n+1]$ is not differential approximate with ratio greater than $\frac{741}{742}$.

We now give some standard definitions:

Definition 1.1 An NPO problem $\pi$ is a five-tuple $(\mathcal{I}$, sol, $m$, Triv, goal) such that:

(i) $\mathcal{I}$ is the set of instances and is recognizable in polynomial-time.

(ii) Given an instance $I \in \mathcal{I}$, sol $[I]$ is the set of feasible solutions of $I$; moreover, there exists a polynomial $P$ such that, for any $x \in \operatorname{sol}[I],|x| \leq P(|I|)$; furthermore, it is decidable in polynomial time whether $x \in \operatorname{sol}[I]$ for any $I$ and for any $x$ such that $|x| \leq P(|I|)$. Finally, there is a feasible solution Triv $(I)^{1}$ computable in polynomial-time for any $I$.

(iii) Given an instance $I$ and a solution $x$ of $I, m[I, x]$ denotes the non-negative integer value of $x$. The function $m$ is computable in polynomial time and is also called the objective function.

(iv) goal $\in\{$ Max, Min $\}$.

We call $\bar{\pi}$ the NPO problem $(\mathcal{I}$, sol, $m$, Triv,$\overline{\text { goal }})$ where $\overline{\text { goal }}$ is defined as follows: if goal = Max, then $\overline{\text { goal }}=$ Min and $\overline{\overline{g o a l}}=$ Max. The goal of an NPO-optimization problem with respect to an instance $I$ is to find an optimum solution $x^{*}$ such that $\operatorname{opt}(I)=m\left[I, x^{*}\right]=\operatorname{goal}\{m[I, x]: x \in \operatorname{sol}[I]\}$. Another important solution of $\pi$ is a worst solution $x_{*}$ defined by: $\operatorname{wor}(I)=m\left[I, x_{*}\right]=\overline{g o a l}\{m[I, x]$ : $x \in \operatorname{sol}[I]\}$. A worst solution for $\pi$ is an optimal solution for $\bar{\pi}$ and vice versa. In Ausiello et al. [4], the term trivial solution referred to as worst solution and all the exposed examples have the property that a worst solution can be trivially computed in polynomial-time. For example, this is the case of the maximum Cut problem where, given a graph, the worst solution is the empty edge-set given by

\footnotetext{
${ }^{1}$ The common definition of class NPO does not require the existence of a trivial solution.
} 
the partition $(V, \emptyset)$, or the Bin-Packing problem where we can trivially put the items using a distinct bin per item. On the contrary, since a worst solution of the maximum weight Hamiltonian path from $s$ to $t$ is an optimal solution of the minimum weight Hamiltonian path from $s$ to $t$, the computation of such a solution is NP-hard. Thus, computing a worst solution of HPP (or $\mathrm{HPP}_{s}$ or $\mathrm{HPP}_{s, t}$ respectively) is as hard as computing an optimal one of $\mathrm{HPP}$ (or $\mathrm{HPP}_{s}$ or $\mathrm{HPP}_{s, t}$ respectively). Note that the same property occurs for a large class of problems, Monnot [27].

\subsection{Approximate algorithms and reductions}

In order to study algorithm performances, there are two known measures: standard ratio [16], [3], [8] and differential ratio [13], [4], [20] and [7].

Definition 1.2 Let $\pi$ be an $N P O$ problem and $x \in \operatorname{sol}[I]$. We define the performance ratios of $x$ with respect to the instance $I$ as

- (standard ratio) $\rho_{\pi}(I, x)=\operatorname{Min}\left\{\frac{m[I, x]}{o p t(I)}, \frac{o p t(I)}{m[I, x]}\right\}$

- (differential ratio) $\delta_{\pi}(I, x)=\frac{w o r(I)-m[I, x]}{\operatorname{wor}(I)-\operatorname{opt}(I)}$

The performance ratio is a number less than or equal to 1 , and is equal to 1 if and only if $m[I, x]=$ opt $(I)$. Note that, compared to some definitions, we have inverted the standard performance ratio in the case of minimization problems so that the ratio value is always between 0 and 1 . Let $\pi$ be an NPO problem. For any instance $I$ of $\pi$, a polynomial time algorithm $A$ returns a feasible solution $x^{A}$. The performance of $A$ with respect to $R \in\{\delta, \rho\}$ on the instance $I$ is the quantity $R_{A}[\pi](I)=R_{\pi}\left(I, x^{A}\right)$. We say that $A$ is an $\varepsilon$-approximation algorithm with respect to $R$ if for any instance $I$, we have $R_{A}(I) \geq \varepsilon$.

Definition 1.3 For any performance ratio $R \in\{\delta, \rho\}$,

- an $\mathbf{N P O}$ problem belongs to the class $\mathbf{A P X}(R)$ if there exists an $\varepsilon$-approximation with respect to $R$ for some constant $\varepsilon \in] 0 ; 1]$.

- an NPO problem belongs to the class $\mathbf{P T A S}(R)$ if there exists an $\varepsilon$-approximation $A_{\varepsilon}$ for any constant $\varepsilon \in] 0 ; 1\left[\right.$. The family $\left\{A_{\varepsilon}\right\}_{0<\varepsilon<1}$ is said to be a polynomial time approximation scheme. $\diamond$

Clearly, the following inclusion holds for any measure $R \in\{\delta, \rho\}$ : $\mathbf{P T A S}(R) \subseteq \mathbf{A P X}(R)$. As it is usually done, we will denote by APX and PTAS, respectively, the classes $\mathbf{A P X}(\rho)$ and $\mathbf{P T A S}(\rho)$. We could argue whether the differential ratio is really pertinent: the authors of [13] and [4] answered positively to that question and concluded that this measure is complementary with the standard ratio. As shown in [11], many problems can have different behavior patterns depending on whether the differential or standard ratio is chosen: consider for instance Vertex Covering or Dominating Set problems. On the other hand, there are problems that establish some connections between the differential and the standard ratios, like Bin Packing [12] or maximum weight bounded-depth spanning tree [28] and see Zemel [36] for motivations and complementarity links between the two 
measures. Besides, we show that there are tight links between both measures for the problems dealt with in the case where the edge-weights have lower and upper bounds.

Now, consider the following approximation preserving reductions between pairs $(\pi, R)$.

Definition 1.4 For $\pi_{i} \in N P O$ and $R_{i} \in\{\delta, \rho\}, i=1,2$,

- an A-reduction from $\left(\pi_{1}, R_{1}\right)$ to $\left(\pi_{2}, R_{2}\right)$, denoted by $\left(\pi_{1}, R_{1}\right) \leq{ }^{A}\left(\pi_{2}, R_{2}\right)$,

is a triplet $(\propto, f, c)$ such that:

(i) $\propto: \mathcal{I}_{\pi_{1}} \longmapsto \mathcal{I}_{\pi_{2}}$, transforms an instance of $\pi_{1}$ into an instance of $\pi_{2}$ in polynomial-time.

(ii) $f: \operatorname{sol}_{\pi_{2}}[\propto(I)] \longmapsto$ sol $_{\pi_{1}}[I]$, transforms solutions for $\pi_{2}$ into solutions for $\pi_{1}$ in polynomialtime.

(iii) $c:[0 ; 1] \longmapsto[0 ; 1]$ (called expansion of the A-reduction) is a function satisfying $c^{-1}(0) \subseteq\{0\}$ and $\forall \varepsilon \in[0 ; 1], \forall I \in \mathcal{I}_{\pi_{1}}, \forall x \in \operatorname{sol}_{\pi_{2}}[\propto(I)]: R_{2}\left[\pi_{2}\right](\propto(I), x) \geq \varepsilon \Longrightarrow R_{1}\left[\pi_{1}\right](I, f(x)) \geq c(\varepsilon)$

- an $A * P$-reduction from the pair $\left(\pi_{1}, R_{1}\right)$ to the pair $\left(\pi_{2}, R_{2}\right)$, denoted by $\left(\pi_{1}, R_{1}\right) \leq{ }^{A * P}\left(\pi_{2}, R_{2}\right)$, is an A-reduction from $\left(\pi_{1}, R_{1}\right)$ to $\left(\pi_{2}, R_{2}\right)$ such that the restriction of function $c$ to some interval $[a ; 1]$ is bijective and $c(1)=1(c(0)$ may be non-zero $)$.

An $A$-reduction preserves constant approximation while $A * P$-reduction preserves approximation schemes. They are a natural generalization of those described by Orponen and Mannila [30] and Crescenzi and Panconesi [9].

Definition 1.5 If $\left(\pi_{1}, R_{1}\right) \leq{ }^{A * P}\left(\pi_{2}, R_{2}\right)$ and $\left(\pi_{2}, R_{2}\right) \leq{ }^{A * P}\left(\pi_{1}, R_{1}\right)$ with $c(\varepsilon)=\varepsilon$, we say that $\left(\pi_{1}, R_{1}\right)$ is equivalent to $\left(\pi_{2}, R_{2}\right)$.

The differential ratio measures how the value of an approximate solution $m[I, x]$ is located in the interval between opt $(I)$ and $\operatorname{wor}(I)$. More exactly it is equivalent for a maximization problem to prove $\delta_{\pi}(I, x) \geq \varepsilon$ and $m[I, x] \geq \operatorname{copt}(I)+(1-\varepsilon)$ wor $(I)$. On the other hand, the standard ratio measures (for a maximization problem) how the value of an approximate solution is placed in the interval between 0 and $\operatorname{opt}(I)$. Hence, we have an $A * P$-reduction from the standard ratio to the differential ratio:

Lemma 1.6 If $\pi=(\mathcal{I}$, sol, $m$, Triv, Max $) \in \boldsymbol{N P O}$, then $(\pi, \rho) \leq{ }^{A * P}(\pi, \delta)$ with $c(\varepsilon)=\varepsilon$.

Proof : Let $I$ be an instance of $\pi$ and $x$ be a feasible solution. If $m[I, x] \geq \operatorname{copt}(I)+(1-\varepsilon)$ wor $(I)$ then we have all the more so $m[I, x] \geq \operatorname{\varepsilon opt}(I)$ since $\operatorname{wor}(I) \geq 0$.

Note that, in general, there is no evident transfer of a positive or negative result from one framework to the other for a minimization problem. For instance, we have proved in Demange et al. [10] that a version of weighted minimum coloring admits a standard non-approximation threshold equal to $\frac{7}{8}$ in bipartite graphs whereas we have built a differential approximation scheme; in this coloring version, the cost of a stable set is given by the maximum of the vertex weights in this stable set. 


\section{The Hamiltonian path problem}

The Hamiltonian path problem, also called the Traveling Salesman Path problem, is formally defined as follows.

Definition 2.1 Consider a complete graph $K_{n}$ with non-negative costs $d(x, y)$ for each vertex pair. We want to find an optimal-cost Hamiltonian path, where the cost of a path is the sum of the weights on its edges. We refer this problem as HPP. When one endpoint s (resp. two endpoints $s$ and $t$ ) of Hamiltonian path are specified, we use the notation $\mathrm{HPP}_{s}\left(\right.$ resp. $\left.\mathrm{HPP}_{s, t}\right)$.

If goal $=$ Max, the problem is called MAX HPP, else MIN HPP. We use notation $\mathrm{HPP}, \mathrm{HPP}_{s}$ or $\mathrm{HPP}_{s, t}$ with no prefix when we consider without distinction the case goal $=$ Max or goal $=$ Min. $\diamond$

Standard ratio approximation results can be derived for HPP by using trivial reduction to TSP: the first negative approximation result (that we can deduce from [33]) states that it is not possible to approximate MiN HPP within $1 / f(|I|)$ where $\mathrm{f}$ is any integer function computable within polynomial time unless $\mathbf{P}=\mathbf{N P}$. On the other hand, metric ${ }^{2}$ Min HPP is approximable within 2/3 [6] and MiN HPP $[1,2]$ is APX-complete (deduced from Papadimitriou and Yannakakis [31]). For MAX HPP, the results are more optimistic since this problem is in APX. The best-known standard ratio is equal to $\frac{25}{33}$ and can be deduced from Hassin and Rubinstein [21].

Min Metric $\operatorname{HPP}_{s, t}$ is as hard to approximate as Min Metric HPP ${ }_{s}$. Is Min Metric $\operatorname{HPP}_{s, t}$ really much harder to approximate than Min METRIC $\operatorname{HPP}_{s}$ ? This interesting question raised the first time by Johnson and Papadimitriou [23] on the relative hardness of the two specified endpoints version compared to the one specified endpoint is still open today. However, the positive results given on these problems lead to a positive answer to the question since the best-known standard ratios are $\frac{2}{3}$ for Min Metric $\operatorname{HPP}_{s}$, Hoogeveen [22] and $\frac{3}{5}$ for Min Metric $\operatorname{HPP}_{s, t}$ Hoogeveen [22], Guttmann-Beck et al. [18]. Finally, if we consider the case $a \leq d(e) \leq 2 a$ there are no specific results. For example Christofides' modification algorithm [22] remains a $2 / 3$-standard ratio for MiN $\operatorname{HPP}_{s}[a ; 2 a]$. To our knowledge, no standard approximation result has been found for MAX $\operatorname{HPP}_{s}$ and MAX $\operatorname{HPP}_{s, t}$.

We show that $\mathrm{HPP}_{s}$ is $\frac{2}{3}$ approximable and $\mathrm{HPP}_{s, t}$ is $\frac{1}{2}$ approximable under the differential framework. We can deduce from Lemma 1.6 a $\frac{2}{3}$-standard approximation for MAX $\mathrm{HPP}_{s}$ and a $\frac{1}{2}$ standard approximation for MAX $\mathrm{HPP}_{s, t}$. Moreover, our technique allows to handle the case where all the edge weights are within an interval $[a, 2 a]$ for any positive $a$ since from previous results, we deduce a $\frac{3}{4}$ (resp. $\frac{2}{3}$ )-standard approximation for $\operatorname{Min}_{\operatorname{HPP}}[a, 2 a]$ (resp. Min $\operatorname{HPP}_{s, t}[a, 2 a]$ ) and a $\frac{5}{6}$ (resp. $\frac{3}{4}$ )-standard approximation for $\operatorname{MAX}_{\operatorname{HPP}_{s}}[a, 2 a]$ (resp. MAX $\operatorname{HPP}_{s, t}[a, 2 a]$ ). Thus for these restrictions, we improve the best-known bounds of $\frac{2}{3}$ (resp. $\frac{3}{5}$ ) for minimization versions given by Hoogeveen [22] (resp. Guttmann-beck et al. [18] or [22]).

\footnotetext{
${ }^{2}$ Satisfying for all vertices $x, y, z$ the inequality: $d(x, y) \leq d(x, z)+d(z, y)$.
} 


\section{$3 \quad$ Elementary properties}

Let us first establish some relations between $\mathrm{HPP}, \mathrm{HPP}_{s}, \mathrm{HPP}_{s, t}$ and different subcases. We prove that $\mathrm{HPP}_{s, t}$ is the most general problem. As a second step, we establish for each problem some connected relations between differential and standard ratios. In the following paragraph, without specification, the properties that we present for $\mathrm{HPP}_{s, t}$ are also true for $\mathrm{HPP}_{s}$ and HPP.

$\mathrm{HPP}_{s, t}$ is as hard as $\mathrm{HPP}_{s}$ (which is itself as hard as HPP) to approximate for both performance ratios. Moreover, from a differential approximability point of view, these different versions are very close to the TSP, even if we consider the restriction $a \leq d(e) \leq b$.

Lemma 3.1 For any goal $\in\{$ Min, Max $\}$, we have:

(i) $($ goal $\operatorname{TSP}[a, b], \delta) \leq^{A * P}\left(\right.$ goal $\left.\operatorname{HPP}_{s, t}[a, b], \delta\right)$ with $c(\varepsilon)=\varepsilon$.

(ii) $($ goal $\operatorname{HPP}[a, b], \delta) \leq^{A * P}($ goal $\operatorname{TSP}[a, b], \delta)$ with $c(\varepsilon)=\varepsilon$.

Proof: We only show the case goal $=$ Max.

- For $(i)$ : Let $I=(n, d)$ with $a \leq d(e) \leq b$ be an instance of MAX TSP $[a, b]$. Choose a vertex $s$ in $K_{n}$ and define $I_{v}=(n, s, v, d)$ an instance of $\operatorname{MAX}_{\operatorname{HPP}_{s, v}}[a, b]$ for every $v \in V \backslash\{s\}$. Let $\mu_{v}$ be a Hamiltonian path from $s$ to $v$ of $I_{v}$ which is an $\varepsilon$-differential approximation for $\operatorname{MAX}_{\operatorname{HPP}} \mathrm{P}_{s, v}[a, b]$. So, for every $v \in V \backslash\{s\}$ we have:

$$
m\left[I_{v}, \mu_{v}\right] \geq \operatorname{copt}_{\mathrm{MAx} \operatorname{HPP}_{s, v}}\left(I_{v}\right)+(1-\varepsilon) \text { wor }_{\mathrm{MAx}_{\mathrm{HPP}}, v}\left(I_{v}\right)
$$

From $\mu_{v}$ with $v \in V \backslash\{s\}$, we construct the Hamiltonian cycle $\Gamma=\operatorname{argmax}\left\{m\left[I, \Gamma_{v}\right]: v \in V \backslash\{s\}\right\}$ where $\Gamma_{v}=\mu_{v} \cup\{(s, v)\}$.

Now, consider $v^{*}$ such that an optimal Hamiltonian cycle of $I=(n, d)$ contains edge $\left(s, v^{*}\right)$; thus, we have:

$$
o p t_{\mathrm{MAx} \operatorname{HPP}_{s, v^{*}}}\left(I_{v^{*}}\right)+d\left(s, v^{*}\right)=o p t_{\mathrm{MAx} \operatorname{TSP}}(I)
$$

Let $\mu_{*}$ be a worst Hamiltonian path from $s$ to $v^{*} ; \mu_{*} \cup\left\{\left(s, v^{*}\right)\right\}$ is an Hamiltonian cycle and we deduce:

$$
\text { wor }_{\operatorname{MAx} \operatorname{HPP}_{s, v^{*}}}\left(I_{v^{*}}\right)+d\left(s, v^{*}\right) \geq \text { wor }_{\mathrm{MAx}} \operatorname{TSP}(I)
$$

Combining inequalities (3.1), (3.2) and (3.3), we obtain: $m[I, \Gamma] \geq m\left[I, \mu_{v^{*}}\right]+d\left(s, v^{*}\right) \geq$ $\operatorname{\varepsilon opt}_{\mathrm{MAX}} \mathrm{TSP}(I)+(1-\varepsilon)$ wor $_{\mathrm{MAx}} \mathrm{TSP}(I)$.

- For (ii): Let $I=(n, d)$ with $a \leq d(e) \leq b$ be an instance of MAX $\operatorname{HPP}[a, b]$. We transform $I$ into instance $\propto(I)=\left(n+1, d^{\prime}\right)$ as follow: add a new vertex $s$ to graph $K_{n}$ and define $d^{\prime}(s, v)=a, \forall v$, $d^{\prime}(e)=d(e)$ for other edges.

Observe that the proof of item $(i i)$ also holds for the standard ratio with goal $=$ Max, but in this case, we might have $a=0$. So, we deduce from the result of Hassin and Rubinstein [21] for MAX 
TSP that MAX HPP is $\frac{25}{33}$-standard approximable. On the other hand, from the result of Sahni and Gonzalez [33] we know that Min $\mathrm{HPP}_{s, t}$ is not in $\mathbf{A P X}$ unless $\mathbf{P}=\mathbf{N P}$. This asymmetry in the approximability of both versions $\left(\mathrm{MAX} \mathrm{HPP}_{s, t}\right.$ is in $\mathbf{A P X}$ as later proved) can be considered as somewhat strange given the structural symmetry existing between them. Since differential approximation is stable under affine transformation of the objective function (see for instance Hassin and Khuller [20] or Demange and Paschos [13]), MAX HPP $s, t$ and Min $\operatorname{HPP}_{s, t}$ are differential-equivalent (see Definition 1.5).

Proposition 3.2 The following assertions hold:

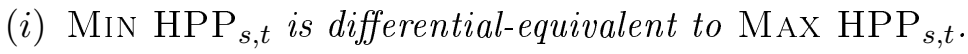

(ii) $\operatorname{MinHPP} P_{s, t}[a, b]$ is differential-equivalent to $\operatorname{MaxHPP} P_{s, t}[a, b]$.

(iii) $\mathrm{HPP}_{s, t}$ is differential-equivalent to metric $\mathrm{HPP}_{s, t}$.

(iv) $\operatorname{HPP}_{s, t}[a, b]$ is differential-equivalent to $\mathrm{HPP}_{s, t}[a+t, b+t]$, for any $t$.

Proof : Let $d_{\max }=\max _{e \in E} d(e)$ and $d_{\min }=\min _{e \in E} d(e)$. Given an instance with distance function $d$ of the left problem in items $(i)-(i v)$, we construct a distance function $d^{\prime}$ to an instance of the corresponding right problem in items $(i)-(i v)$ as follows: $(i) d^{\prime}(e)=d_{\max }+d_{\min }-d(e),(i i)$ $d^{\prime}(e)=a+b-d(e),($ iii $) d^{\prime}(e)=d_{\max }+d(e),(i v) d^{\prime}(e)=t+d(e)$. Since differential ratio is stable under affine transformation of the objective function (see Demange and Paschos [13] or Hassin and Khuller [20]), this concludes the proof.

Observe that the $(i v)$ of this proposition allows to deal with the case where the distances are negative. The following easy theorem holds, thus giving a bridge between differential and standard ratios for $g o a l=M a x$ and goal $=$ Min, in the case where edge weights belong to an interval $[a, b]$.

Theorem 3.3 (goal $\left.\mathrm{HPP}_{s, t}[a, b], \rho\right) \leq^{A * P}\left(\right.$ goal $\left.\mathrm{HPP}_{s, t}[a, b], \delta\right)$ with the expansion satisfying:

$$
\begin{aligned}
& \text { - } c_{1}(\varepsilon)=\frac{(b-a) \varepsilon}{b}+\frac{a}{b} \text { if goal }=\text { Max } \\
& \text { - } c_{2}(\varepsilon)=\frac{a}{b-(b-a) \varepsilon} \text { if goal }=\text { Min }
\end{aligned}
$$

Proof : We only prove the goal $=$ Max case. Let $I$ be an instance and $\mu$ be a Hamiltonian path from $s$ to $t$. If $m[I, \mu] \geq \operatorname{sopt}(I)+(1-\varepsilon) \operatorname{wor}(I)$, then $m[I, \mu] \geq c_{1}(\varepsilon) \operatorname{opt}(I)$ since $\operatorname{wor}(I) \geq \frac{a}{b} \operatorname{opt}(I)$. $\square$

The Proposition 3.2 and the Theorem 3.3 also hold for $\mathrm{HPP}_{s}$ and more generally, these results work for many specific optimization problems from graph theory, those for which all feasible solutions have an equal size that depends on the instance size (see Monnot [27]).

$\operatorname{MAX~HPP}_{s, t}[a, b]$ and MiN $\operatorname{HPP}_{s, t}[a, b]$ (for $a$ and $b$ not depending on the instance) are trivially in $\mathbf{A P X}$ when $a>0$ since any solution is at least a $a / b$-standard approximation (take $\varepsilon=0$ in 
Theorem 3.3); in this case, the standard ratio may not be that meaningful since even a worst solution yields a constant standard approximation. Nevertheless, we can deduce from this theorem that the hardness thresholds for standard and differential framework are identical since MiN $\operatorname{HPP}_{s, t}[a, b]$ is

\section{APX-complete.}

Corollary 3.4 For all $b>a \geq 0, \operatorname{HPP}_{s, t}[a, b] \notin \mathbf{P T A S}(\delta)$ unless $\boldsymbol{P}=\boldsymbol{N P}$.

We can also establish a limit on its differential approximation for some values of $a$ and $b$. Recall the negative result of Engebretsen and Karpinski [14] for MiN TSP[1,2]: for any $\epsilon>0$, no polynomial time algorithm can guarantee a standard approximation ratio greater than, or equal to, $\frac{740}{741}+\epsilon$.

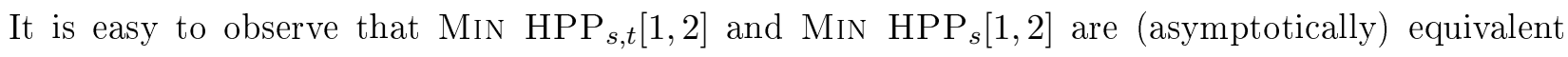
to approximate Min TSP[1,2]. Thus, we can deduce that $\operatorname{Min} \operatorname{HPP}_{s}[1,2]$ and $\operatorname{Min} \operatorname{HPP}_{s, t}[1,2]$ are not standard approximable with ratio greater than $\frac{740}{741}$. Finally, using Theorem 3.3 and $(i v)$ of Proposition 3.2, we obtain:

Proposition 3.5 For all $a, \mathrm{HPP}_{s, t}[a, a+1]$ and $\operatorname{HPP}_{s}[a, a+1]$ are not approximable with differential ratio greater than $\frac{741}{742}$ unless $\boldsymbol{P}=\boldsymbol{N P}$.

\section{Approximate algorithms for these problems}

In this section, we propose two types of algorithms which yield constant differential-ratio. For MAX $\mathrm{HPP}_{s}$, the algorithm is obtained by getting several feasible solutions and by choosing the best one among them. Each of these individual solutions has a differential approximation ratio tending towards zero with the size of the instance. For MAX $\operatorname{HPP}_{s, t}$, the algorithm is very different and takes into account the extreme solutions. So, on the one hand, the algorithm tries to be the nearest from the best solution value and on the other hand, tries to be the furthest from the worst solution value. In order to do that, it iteratively provides a solution of value greater than $\left(w o r\left(I_{j}\right)+\operatorname{opt}\left(I_{j}\right)\right) / 2$, where $I_{j}$ is the sub-graph built at step $j$.

\subsection{The algorithm for two specified endpoints version}

MAX $\mathrm{HPP}_{s, t}$ can also be regarded as the problem of determining a Hamiltonian cycle that contains edge $(s, t)$. The algorithm works by finding a maximum weight 2-matching among 2-matchings containing $(s, t)$ and at each step, merging the cycles two by two. The main idea consists in pointing out that we could have lost much more by merging the two cycles in a different way. Thus, we will build dynamically another solution which approximate the worst solution; this solution will actually depends on the choices made by the algorithm at each iteration.

Consider two cycles $C_{i}$ and two edges $\left(x_{1}, x_{2}\right) \in C_{1}$ and $\left(y_{1}, y_{2}\right) \in C_{2}$, we call localchange lor $_{i}$ $i=1,2$ the following process:

$$
\text { localchange }_{i}\left[\left(C_{1},\left(x_{1}, x_{2}\right)\right),\left(C_{2},\left(y_{1}, y_{2}\right)\right)\right]=\left\{\left(x_{1}, y_{3-i}\right),\left(x_{2}, y_{i}\right)\right\} \cup\left(C_{1} \cup C_{2} \backslash\left\{\left(x_{1}, x_{2}\right),\left(y_{1}, y_{2}\right)\right\}\right)
$$


These two processes merge the cycles $C_{1}$ and $C_{2}$ into a single cycle (see the Figure 1 for an illustration.). Note that the vertex order is important in the processes; thus, edges $\left(x_{1}, x_{2}\right)$ or $\left(y_{1}, y_{2}\right)$

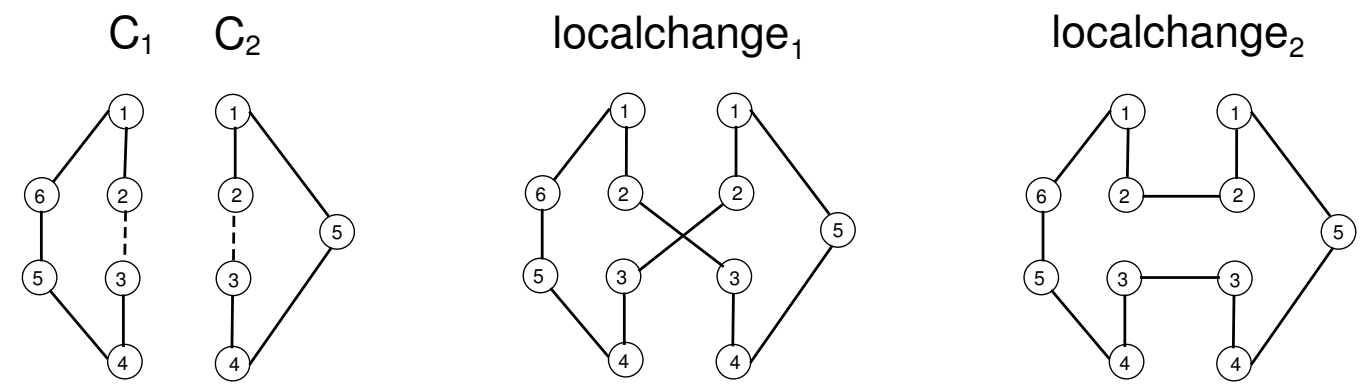

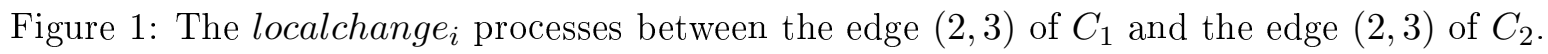

are implicitly given as directed edges and we have: localchange $1\left[\left(C_{1},\left(x_{2}, x_{1}\right)\right),\left(C_{2},\left(y_{1}, y_{2}\right)\right)\right]=$ localchange $_{2}\left[\left(C_{1},\left(x_{1}, x_{2}\right)\right),\left(C_{2},\left(y_{1}, y_{2}\right)\right)\right]$. Moreover, when $C_{1}=C_{2}$ and $\left(x_{1}, x_{2}\right)$ is not adjacent to $\left(y_{1}, y_{2}\right)$, these processes simply amount to local edge swaps. We associate with localchange $_{i}$ a function cost $_{i}$ that represents the loss in merging two cycles:

$$
\operatorname{cost}_{i}\left[\left(x_{1}, x_{2}\right),\left(y_{1}, y_{2}\right)\right]=d\left(x_{1}, x_{2}\right)+d\left(y_{1}, y_{2}\right)-d\left(x_{1}, y_{3-i}\right)-d\left(x_{2}, y_{i}\right)
$$

[LocalchangeHPP $\left.P_{s, t}\right]$

input: An instance $(n, s, t, d)$;

output: A Hamilton path sol from $s$ to $t$;

Change the cost of $(s, t)$ into $|V| d_{\max }+1$. Call this function $d^{\prime}$;

Compute a maximum weight 2-matching $M=\left\{C_{i}, i=1, \ldots, k\right\}$ of $\left(n, d^{\prime}\right)$;

Suppose that $(s, t) \in C_{1}$

Choose 2 consecutive edges $\left(x_{1}^{1}, x_{2}^{1}\right)$ and $\left(x_{2}^{1}, x_{3}^{1}\right)$ in $C_{1}$ different from $(s, t)$;

sol $_{1}=C_{1} \backslash\{(s, t)\}, e_{1}^{1}=\left(x_{1}^{1}, x_{2}^{1}\right)$ and $e_{2}^{1}=\left(x_{2}^{1}, x_{3}^{1}\right)$;

For $\mathrm{i}=2$ to $\mathrm{k}$ do

Choose 2 consecutive edges $\left(x_{1}^{i}, x_{2}^{i}\right)$ and $\left(x_{2}^{i}, x_{3}^{i}\right)$ in $C_{i}$;

If $\operatorname{cost}_{1}\left[e_{1}^{i-1},\left(x_{1}^{i}, x_{2}^{i}\right)\right] \leq \operatorname{cost}_{2}\left[e_{2}^{i-1},\left(x_{2}^{i}, x_{3}^{i}\right)\right]$ then

sol $_{i}=$ localchange $_{1}\left[\left(\operatorname{sol}_{i-1}, e_{1}^{i-1}\right),\left(C_{i},\left(x_{1}^{i}, x_{2}^{i}\right)\right)\right]$;

Suppose $e_{1}^{i-1}=(x, y)$, and $x_{0}^{i}$ is the other neighbor of $x_{1}^{i}$ in $C_{i}$

Set $e_{1}^{i}=\left(y, x_{1}^{i}\right)$ and $e_{2}^{i}=\left(x_{1}^{i}, x_{0}^{i}\right)$;

Else

sol $_{i}=$ localchange $_{2}\left[\left(\operatorname{sol}_{i-1}, e_{2}^{i-1}\right),\left(C_{i},\left(x_{2}^{i}, x_{3}^{i}\right)\right)\right]$;

Suppose $e_{2}^{i-1}=(x, y)$, and $x_{4}^{i}$ is the other neighbor of $x_{3}^{i}$ in $C_{i}$

Set $e_{1}^{i}=\left(y, x_{3}^{i}\right)$ and $e_{2}^{i}=\left(x_{3}^{i}, x_{4}^{i}\right)$;

End if ;

End for i ;

sol $=\operatorname{sol}_{k}$; 
As this algorithm is polynomial, let us then show that sol is an Hamiltonian path. Firstly, note that by construction, $(s, t)$ belongs to every maximum weight 2-matching of $\left(n, d^{\prime}\right)$. Moreover, $e_{1}^{i}$ and $e_{2}^{i}$ obviously belong to $\operatorname{sol}_{i}$ for every iteration $i \leq k$ of the algorithm. These two facts lead to the result. A description of the algorithm is given in the Figure 2 when $M=\left\{C_{i}: i=1,2,3\right\}$ with $\left|C_{1}\right|=6$, $\left|C_{2}\right|=4$ and $\left|C_{2}\right|=5$.
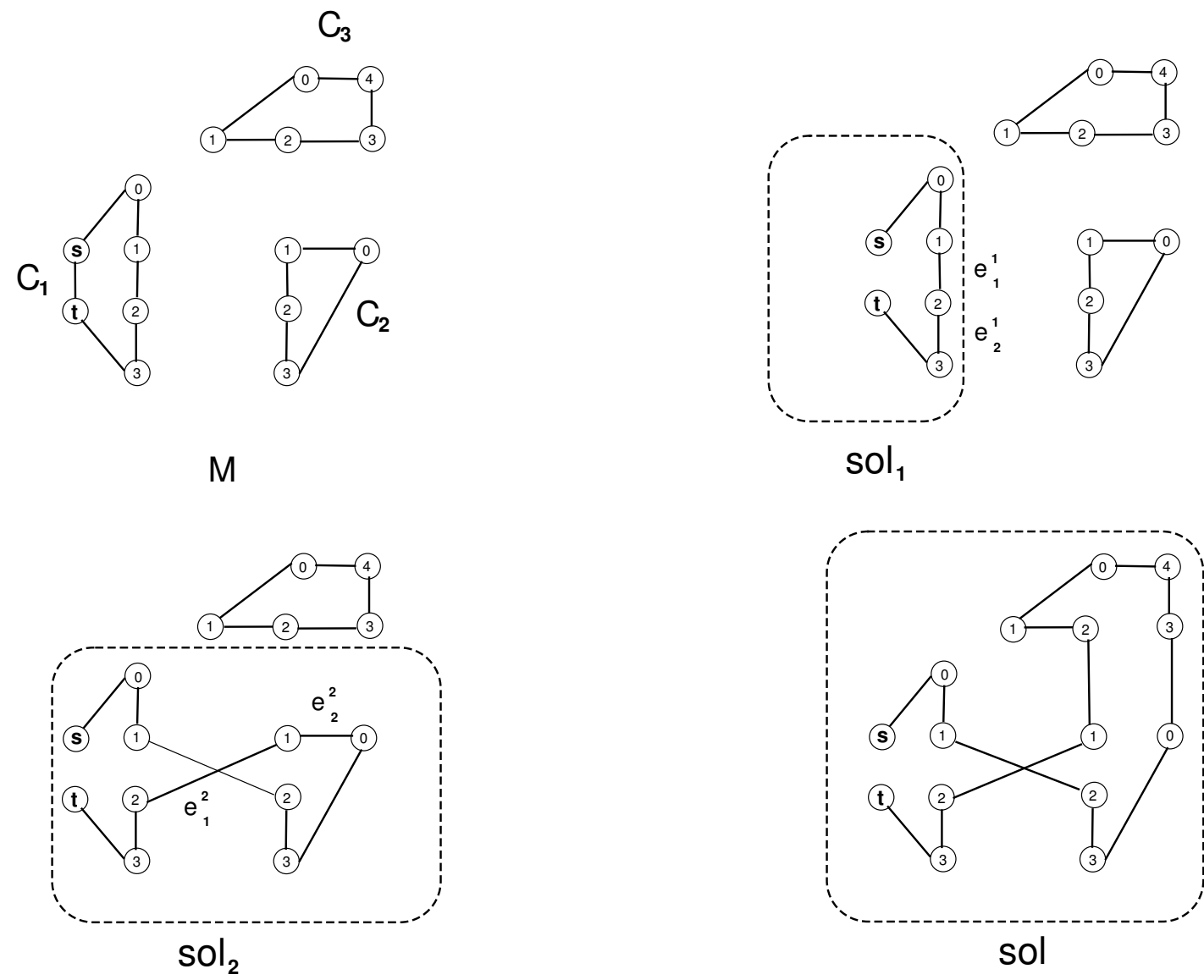

Figure 2: The 2-Matching $M$ and the different iterations of algorithm when $k=3$.

Theorem 4.1 The algorithm [LocalchangeHPP $P_{s, t}$ ] is a $\frac{1}{2}$-differential approximation for MAX $\mathrm{HPP}_{s, t}$ and this ratio is tight.

Proof: Given $I=(n, s, t, d)$, an instance of $\operatorname{MAx} \operatorname{HPP}_{s, t}$, we denote $\left(i_{2}, \ldots, i_{k}\right)$ with $i_{j} \in\{1,2\}$ the sequence of choices produced by the algorithm such that, for $j \in\{2, \ldots, k\}$ :

$$
\operatorname{sol}_{j}=\text { localchange }_{i_{j}}\left[\left(\operatorname{sol}_{j-1}, e_{i_{j}}^{j-1}\right),\left(C_{j},\left(x_{i_{j}}^{j}, x_{i_{j}+1}^{j}\right)\right)\right]
$$

Thus, $d\left(\operatorname{sol}_{j}\right)=d\left(\operatorname{sol}_{j-1}\right)+d\left(C_{j}\right)-\operatorname{cost}_{i_{j}}(j)$ with $\operatorname{cost}_{i_{j}}(j)=\operatorname{cost}_{i_{j}}\left[e_{i_{j}}^{j-1},\left(x_{i_{j}}^{j}, x_{i_{j}+1}^{j}\right)\right]$. Summing 
up these equalities for $j=2$ to $k$, and since $d\left(\operatorname{sol}_{1}\right)=d\left(C_{1}\right)-d(s, t)$ and $d\left(\right.$ sol $_{)}=d\left(\right.$ sol $\left._{k}\right)$, we obtain:

$$
d(s o l)=d(M)-d(s, t)-\sum_{j=2}^{k} \operatorname{cost}_{i_{j}}(j)
$$

The main idea is to note that edge-subset $\left\{e_{3-i_{j}}^{j-1},\left(x_{3-i_{j}}^{j}, x_{4-i_{j}}^{j}\right): j=2, \ldots, k\right\}$ belongs to solution sol $_{k}$. Hence, we can "damage" the current solution by local edges-swap from this edge-subset. More formally, consider solutions $\operatorname{sol}_{j}^{\prime}$ defined by $\operatorname{sol}_{1}^{\prime}=\operatorname{sol}_{k}$ and for $j=2, \ldots, k$,

$$
\text { sol }_{j}^{\prime}=\text { localchange }_{3-i_{j}}\left[\left(\operatorname{sol}_{j-1}^{\prime}, e_{3-i_{j}}^{j-1}\right),\left(\operatorname{sol}_{j-1}^{\prime},\left(x_{3-i_{j}}^{j}, x_{4-i_{j}}^{j}\right)\right)\right]
$$

An illustration of solutions $s^{\prime} l_{i}^{\prime}$ with $i \leq k$ is depicted in the Figure 3 for the example described in Figure 2.
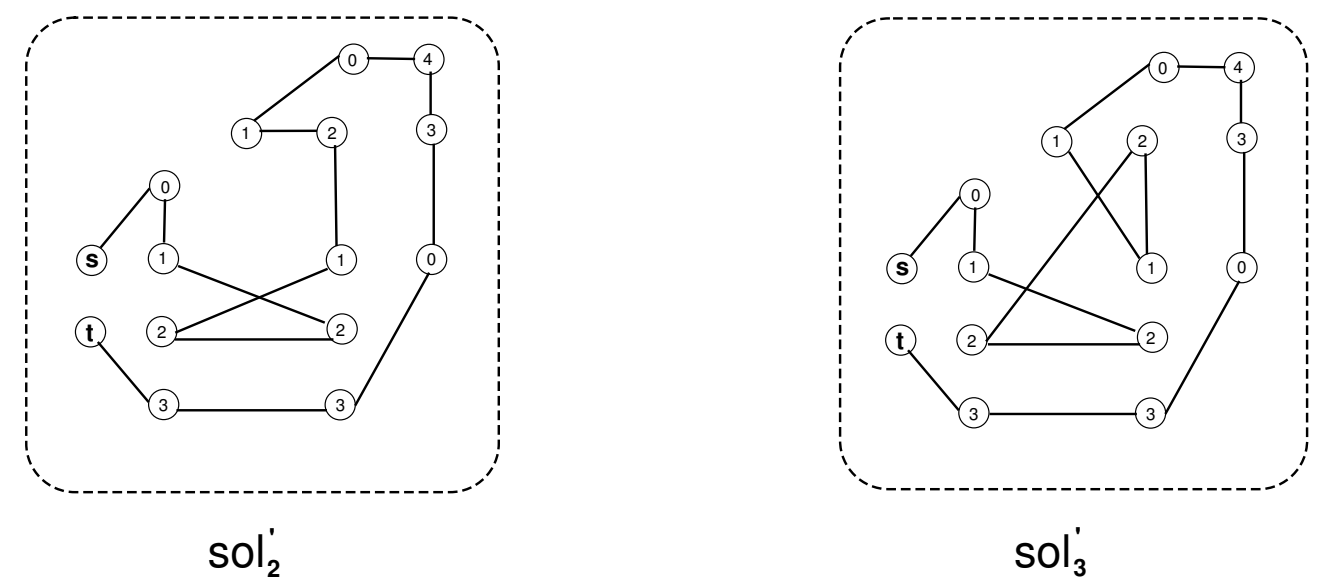

Figure 3: The solutions $\operatorname{sol}_{2}^{\prime}$ and $\operatorname{sol}_{3}^{\prime}$.

Lastly, proceeding as previously, we obtain $d\left(\operatorname{sol}_{k}^{\prime}\right)=d(M)-d(s, t)-\sum_{j=2}^{k}\left(\operatorname{cost}_{i_{j}}(j)+\operatorname{cost}_{3-i_{j}}(j)\right)$. By construction, $\operatorname{cost}_{i_{j}}(j)+\operatorname{cost}_{3-i_{j}}(j) \geq 2 \operatorname{cost}_{i_{j}}(j)$ and wor $_{(I)} \leq d\left(\operatorname{sol}_{k}^{\prime}\right)$. Hence:

$$
w o r(I) \leq d(M)-d(s, t)-2 \sum_{j=2}^{k} \operatorname{cost}_{i_{j}}(j)
$$

$M$ is an optimal weight 2-matching among the 2-matching of $(n, d)$ containing the edge $(s, t)$; thus

$$
\operatorname{opt}(I) \leq d(M)-d(s, t)
$$

By combining expressions (4.3),(4.2) and (4.1), we obtain:

$$
d(s o l) \geq \frac{1}{2} \operatorname{opt}(I)+\frac{1}{2} \operatorname{wor}(I)
$$

We now show that this ratio is tight. Let $J_{n}=(n, s, t, d)$ be an instance defined by: $V=$ $\left(\left\{x_{i}^{j}, 1 \leq i \leq 3,2 \leq j \leq 2 n+1\right\} \cup\{s, u, t\}\right), d\left(x_{1}^{j}, x_{1}^{j+1}\right)=d\left(x_{1}^{j}, x_{2}^{j+1}\right)=1 \forall j=2, \ldots, 2 n$, 
$d\left(x_{1}^{j}, x_{2}^{j+2}\right)=1 \forall j=2, \ldots, 2 n-1, d_{n}\left(s, x_{2}^{2}\right)=d_{n}\left(u, x_{1}^{2}\right)=d\left(u, x_{2}^{2}\right)=d\left(t, x_{3}^{2}\right)=1$ and let the cost of all other edges be two. The 2-matching is composed of $C_{1}=\{s, u, t\}$ and $C_{j}=\left\{x_{1}^{j}, x_{2}^{j}, x_{3}^{j}\right\} j=$ $2, \ldots, 2 n+1$. The edges produced by the algorithm are: $e_{1}^{1}=(s, u), e_{2}^{1}=(u, t), e_{2}^{1}=\left(u, x_{1}^{2}\right), e_{2}^{2}=$ $\left(x_{1}^{2}, x_{3}^{2}\right), e_{1}^{j}=\left(x_{1}^{j-1}, x_{1}^{j}\right), e_{2}^{j}=\left(x_{1}^{j}, x_{3}^{j}\right) j=3, \ldots, 2 n+1$ and $\operatorname{cost}_{1}(2)=\operatorname{cost}_{2}(2)=2, \operatorname{cost}_{1}(j)=$ $\operatorname{cost}_{2}(j)=1 j=3, \ldots, 2 n+1$.

$$
d(\operatorname{sol})=10 n+4, \operatorname{wor}\left(J_{2 n+1}\right)=8 n+3, \quad \operatorname{opt}\left(J_{2 n+1}\right)=12 n+4
$$

Thus, we obtain that $\delta_{\text {LocalchangeHPP }}\left(J_{2 n+1}\right)$ approaches $\frac{1}{2}$ as $n$ goes to infinity.

For the standard ratio, we deduce two new improved results by using Lemma 1.6 from the general case and Theorem 3.3 with $b=2 a$ for the case where the weights of the graph are bounded between the values $a$ and $2 a$.

Corollary 4.2 We have the following results:

- $\mathrm{MAX} \mathrm{HPP}_{s, t}$ is $\frac{1}{2}$-standard approximable and Max $H P P_{s, t}[a, 2 a]$ is $\frac{3}{4}$-standard approximable.

- $\operatorname{Min} \operatorname{HPP}_{s, t}[a, 2 a]$ is $\frac{2}{3}$-standard approximable.

\subsection{The algorithm for one specified endpoint version}

We propose an algorithm which differs from the one previously studied since we explicitly compute several solutions. Our algorithm is based upon a simple idea and uses structural properties of solutions. It still works by finding a maximum weight 2-matching containing specified edges and then discarding some edges and arbitrarily connecting the resulting paths to form an Hamiltonian path from $s$. The principle of our algorithm is to generate not only one but several feasible solutions following this method.

Consider a maximum weight 2-matching $M_{r}$ among those containing $(s, r)$, including elementary cycles $C_{i}, i=1, \ldots, k$. In order to do that, we substitute $|V| d_{\max }+1$ for the cost of $(s, r)$ and we compute a maximum 2-matching in this new instance. Lastly, for each cycle $C_{i}$, we consider four consecutive vertices $x_{1}^{i}, x_{2}^{i}, x_{3}^{i}, x_{4}^{i}$. Note that we have numbered vertices such that $x_{1}^{1}=r$ and $x_{2}^{1}=s$. Moreover, if $\left|C_{i}\right|=3$ then $x_{4}^{i}=x_{1}^{i}$. For the last cycle $C_{k}$, we consider an additional vertex $y$ which is the other neighbor of $x_{1}^{k}$ in $C_{k}$. Thus, if $\left|C_{k}\right|=4$ then $y=x_{4}^{k}$ while $y$ is a new vertex in the other case.

[Patching 2-matching]

input: An instance $(n, s, d)$;

output: A Hamiltonian path sol from $s$;

For every $r \in V \backslash\{s\}$ do

Change the cost of $(s, r)$ into $|V| d_{\max }+1$. Call this function $d^{\prime}$;

Compute a maximum weight 2-matching $M_{r}=\left\{C_{i}, i=1, \ldots, k\right\}$ of $\left(n, d^{\prime}\right)$;

if $k=1$ then sol $_{r}=M_{r} \backslash\{(s, r)\}$;

if $k$ is even then 
$S_{1}=\cup_{j=1}^{k-1}\left\{\left(x_{2}^{j}, x_{3}^{j}\right)\right\} \cup\left\{\left(x_{1}^{k}, x_{2}^{k}\right),(s, r)\right\} ;$

Build $\operatorname{sol}_{1}=\left(M_{r} \backslash S_{1}\right) \cup\left\{\left(x_{1}^{k}, x_{3}^{1}\right),\left(x_{2}^{1}, x_{2}^{2}\right)\right\} \cup_{j=1}^{(k-2) / 2}\left\{\left(x_{3}^{2 j}, x_{3}^{2 j+1}\right),\left(x_{2}^{2 j+1}, x_{2}^{2 j+2}\right)\right\}$;

( $s o l_{1}$ is a Hamiltonian path from $s$ to $r$ )

$S_{2}=\cup_{j=2}^{k-1}\left\{\left(x_{1}^{j}, x_{2}^{j}\right)\right\} \cup\left\{\left(y, x_{1}^{k}\right),(s, r)\right\}$;

Build sol $_{2}=\left(M_{r} \backslash S_{2}\right) \cup\left\{\left(x_{1}^{1}, x_{1}^{2}\right)\right\} \cup_{j=1}^{(k-2) / 2}\left\{\left(x_{2}^{2 j}, x_{2}^{2 j+1}\right),\left(x_{1}^{2 j+1}, x_{1}^{2 j+2}\right)\right\}$;

$\left(\mathrm{sol}_{2}\right.$ is a Hamiltonian path from $s$ to $y$ )

$S_{3}=\cup_{j=1}^{k-1}\left\{\left(x_{3}^{j}, x_{4}^{j}\right)\right\} \cup\left\{\left(x_{2}^{k}, x_{3}^{k}\right),(s, r)\right\} ;$

Build $s_{0}=\left(M_{r} \backslash S_{3}\right) \cup\left\{\left(x_{2}^{k}, x_{4}^{1}\right),\left(x_{3}^{1}, x_{3}^{2}\right)\right\} \cup_{j=1}^{(k-2) / 2}\left\{\left(x_{4}^{2 j}, x_{4}^{2 j+1}\right),\left(x_{3}^{2 j+1}, x_{3}^{2 j+2}\right)\right\}$;

$\left(\mathrm{sol}_{3}\right.$ is an Hamiltonian path from $s$ to $r$ )

End if ;

if $k$ is odd then

$S_{1}=\cup_{j=1}^{k}\left\{\left(x_{2}^{j}, x_{3}^{j}\right)\right\} \cup\{(s, r)\} ;$

Build $\operatorname{sol}_{1}=\left(M_{r} \backslash S_{1}\right) \cup\left\{\left(x_{2}^{k}, x_{3}^{1}\right)\right\} \cup_{j=1}^{(k-1) / 2}\left\{\left(x_{2}^{2 j-1}, x_{2}^{2 j}\right)\left(x_{3}^{2 j}, x_{3}^{2 j+1}\right)\right\}$;

$\left(\operatorname{sol}_{1}\right.$ is an Hamiltonian path from $s$ to $r$ )

$S_{2}=\{(s, r)\} \cup_{j=2}^{k}\left\{\left(x_{1}^{j}, x_{2}^{j}\right)\right\}$;

Build $s o l_{2}=\left(M_{r} \backslash S_{2}\right) \cup_{j=1}^{(k-1) / 2}\left\{\left(x_{1}^{2 j-1}, x_{1}^{2 j}\right),\left(x_{2}^{2 j}, x_{2}^{2 j+1}\right)\right\}$;

$\left(\mathrm{sol}_{2}\right.$ is a Hamiltonian path from $s$ to $\left.x_{1}^{k}\right)$

$S_{3}=\cup_{j=1}^{k}\left\{\left(x_{3}^{j}, x_{4}^{j}\right)\right\} \cup\{(s, r)\}$;

Build $s o l_{3}=\left(M_{r} \backslash S_{3}\right) \cup\left\{\left(x_{3}^{k}, x_{4}^{1}\right)\right\} \cup_{j=1}^{(k-1) / 2}\left\{\left(x_{3}^{2 j-1}, x_{3}^{2 j}\right),\left(x_{4}^{2 j}, x_{4}^{2 j+1}\right)\right\}$;

$\left(\mathrm{sol}_{3}\right.$ is a Hamiltonian path from $s$ to $r$ )

End if ;

$\operatorname{sol}_{r}=\operatorname{argmax}\left\{d\left(\operatorname{sol}_{1}\right), d\left(\operatorname{sol}_{2}\right), d\left(\operatorname{sol}_{3}\right)\right\} ;$

End for $\mathrm{r}$;

sol $\left.=\operatorname{argmax}_{d}\left(\operatorname{sol}_{r}\right): r \in V \backslash\{s\}\right\} ;$

Observe that for every $r$, the solutions $s_{1} l_{1}, s_{2} l_{2}$ and $s_{3} l_{3}$ are Hamiltonian paths (from $s$ to different endpoints) since the additional edges are adjacent to the ones substituted. A description of solutions $\operatorname{sol}_{1}$, sol $_{2}$, sol $_{3}$ is given in the Figure 4 when $M_{r}=\left\{C_{i}: i=1,2,3\right\}$ with $\left|C_{1}\right|=\left|C_{3}\right|=6$ and $\left|C_{2}\right|=3$.

The time-complexity of this algorithm remains polynomial since the computation of the 2matching problem is polynomial.

Theorem 4.3 The algorithm [Patching 2-matching] is a $\frac{2}{3}$-differential approximation for MAX $\mathrm{HPP}_{s}$ and this ratio is tight.

Proof: Let $I=(n, s, d)$ be an instance and let $s o l^{*}$ be an optimal Hamiltonian path from $s$ to $r^{*}$. We denote $\operatorname{loss}_{i}, \quad i=1,2,3$, the quantity $d\left(\operatorname{sol}_{i}\right)-d\left(M_{r^{*}}\right)+d\left(s, r^{*}\right)$. Obviously, loss $_{i} \leq 0$ and we have

$$
d(\operatorname{sol}) \geq d\left(\operatorname{sol}_{r}^{*}\right) \geq d\left(M_{r^{*}}\right)-d\left(s, r^{*}\right)+\frac{1}{3}\left(\operatorname{loss}_{1}+\operatorname{loss}_{2}+\operatorname{loss}_{3}\right)
$$

Moreover, the following structural property holds:

$$
\text { sol }_{*}=\cup_{j=1,2,3}\left(\operatorname{sol}_{j} \backslash M_{r^{*}}\right) \cup M_{r^{*}} \backslash\left(S_{1} \cup S_{2} \cup S_{3}\right) \text { is a Hamiltonian path starting from } s
$$



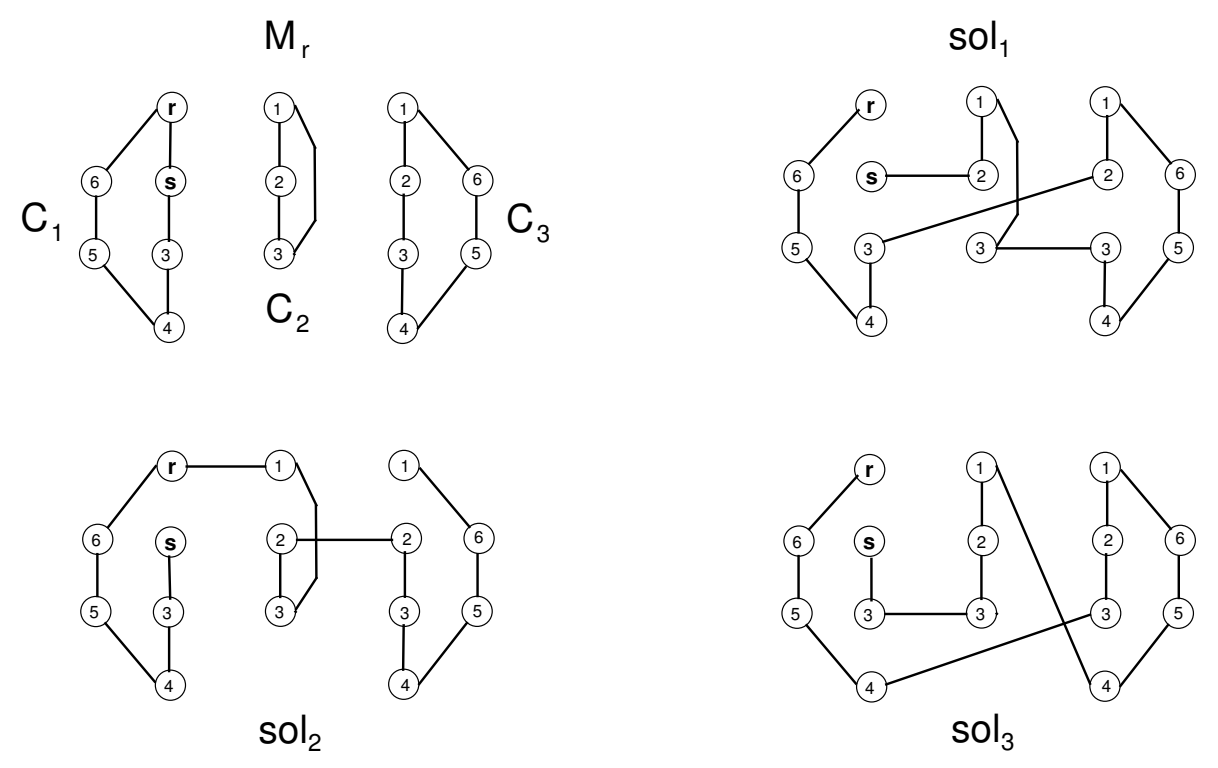

Figure 4: The 2-Matching $M_{r}$ and the solutions $\operatorname{sol}_{1}$, sol $_{2}$ and $\operatorname{sol}_{3}$ when $k=3$.

A description of solution $s_{*} l_{*}$ is depicted in the Figure 5 for the example described in Figure 4.

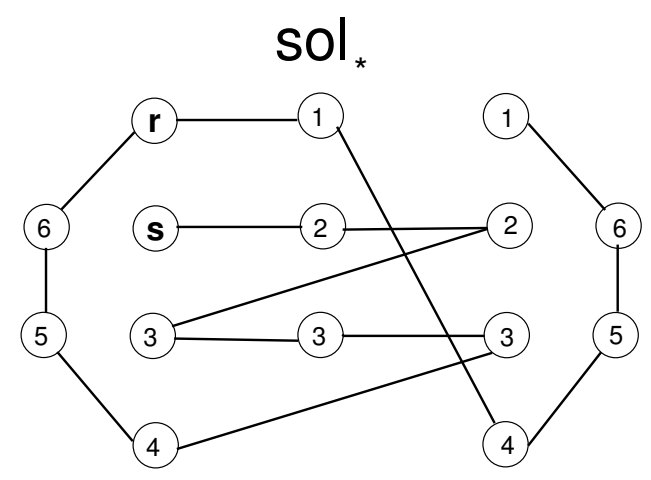

Figure 5: The solution $\operatorname{sol}_{*}$.

$$
d\left(\operatorname{sol}_{*}\right)=d\left(M_{r^{*}}\right)-d\left(s, r^{*}\right)+\operatorname{loss}_{1}+\operatorname{loss}_{2}+\text { loss }_{3} \text { since } d\left(\text { sol }_{j} \backslash M_{r^{*}}\right)=l_{\text {loss }}+d\left(S_{j}\right)-d\left(s, r^{*}\right)
$$
and $d\left(M_{r^{*}} \backslash\left(S_{1} \cup S_{2} \cup S_{3}\right)\right)=d\left(M_{r^{*}}\right)-d\left(S_{1}\right)-d\left(S_{2}\right)-d\left(S_{3}\right)+2 d\left(s, r^{*}\right)$. Hence, we deduce

$$
\operatorname{wor}(I) \leq d\left(M_{r^{*}}\right)-d\left(s, r^{*}\right)+\operatorname{loss}_{1}+\operatorname{loss}_{2}+\operatorname{loss}_{3}
$$

Since $s o l^{*} \cup\left(s, r^{*}\right)$ is a particular 2-matching containing $\left(s, r^{*}\right)$, we have:

$$
\operatorname{opt}(I) \leq d\left(M_{r^{*}}\right)-d\left(s, r^{*}\right)
$$

Lastly, combining (4.4),(4.5) and (4.6) we obtain: 


$$
d(\operatorname{sol}) \geq \frac{1}{3} w o r(I)+\frac{2}{3} \operatorname{opt}(I)
$$

To show that the bound is approachable, consider the following instances. Let $I_{n}=(n, s, d)$ be an instance defined by: $V=\left\{x_{i}^{j}: 1 \leq i \leq 3,1 \leq j \leq 2 n+1\right\}$ with $x_{2}^{1}=s, d\left(x_{1}^{j}, x_{2}^{j}\right)=d\left(x_{3}^{j}, x_{2}^{j}\right)=$ $d\left(x_{1}^{j}, x_{3}^{j}\right)=2, \forall j=1, \ldots, 2 n+1, d\left(x_{1}^{j}, x_{3}^{j+1}\right)=2, \forall j=1, \ldots, 2 n$ and $d\left(x_{1}^{1}, x_{3}^{j}\right)=d\left(x_{1}^{1}, x_{2}^{j}\right)=$ $2, \forall j=2, \ldots, 2 n+1$. Let the cost of all other edges be one. We have:

$$
d(\operatorname{sol}) \leq 10 n+4, \operatorname{opt}\left(I_{n}\right)=12 n+4, \operatorname{wor}\left(I_{n}\right)=6 n+2
$$

leading to the conclusion that $\delta_{\text {Patching }} 2-$ matching $\left(I_{n}\right)$ approaches $\frac{2}{3}$ as $n$ goes to infinity.

As previously, we deduce two new improved standard approximation results by using Lemma 1.6 from the general case and Theorem 3.3 with $b=2 a$ when $a \leq d(e) \leq 2 a$.

Corollary 4.4 We have the following results:

- $\mathrm{MAX} \mathrm{HPP}_{s}$ is $\frac{2}{3}$-standard approximable and $\mathrm{MAX} \mathrm{HPP}_{s}[a, 2 a]$ is $\frac{5}{6}$-standard approximable.

- $\operatorname{Min} \operatorname{HPP}_{s}[a, 2 a]$ is $\frac{3}{4}$-standard approximable.

\section{$5 \quad$ Conclusion and open problems}

In this paper, we have mainly provided new results concerning the approximability of the Hamiltonian path problem in the case in which one or two endpoints are specified. Moreover, we have exposed some basic properties (mainly by using reductions preserving differential approximation) between these problems and some variants of them.

Although in introduction we have pointed out some great difference between the differential and standard approximability of these problems, when we use bounded metric and especially, when the weights in the graph are between the values $a$ and $2 a$, from differential approximation results we can derive new standard approximation results.

An interesting open problem under differential framework is to know if the two-specified endpoints version is really more difficult to approximate than the one-specified endpoint version (we only know that $\mathrm{HPP}_{s, t}$ is at least as hard as $\mathrm{HPP}_{s}$ ). This question is still open under standard framework. The positive approximation results indicate a positive answer but it is not a formal proof. A formal proof would show that the differential non-approximation threshold for $\mathrm{HPP}_{s, t}$ is strictly better than the differential non-approximation threshold for $\mathrm{HPP}_{s}$. In order to prove that, a useful technique is to prove that a problem is not simple. Recall that an NPO problem is called simple by Paz and Moran [32] if its restriction to instances satisfying for any fixed integer $k, \operatorname{opt}(I) \leq k$, can be resolved within polynomial time. So, we can also prove a standard non-approximation threshold equal to $\frac{2}{3}$ for the Bin-Packing problem because its restriction to instances verifying $\operatorname{opt}(I) \leq 2$ is still a NP-hard problem. Similarly, we will say that $\pi$ is $\delta$-simple if its restriction $\pi_{k}$ to instances verifying for any integer fixed $k,|\operatorname{wor}(I)-\operatorname{opt}(I)| \leq k$ can be solved in polynomial time. Thus, if the sub-problem verifying $|\operatorname{wor}(I)-\operatorname{opt}(I)| \leq k_{0}$ is NP-hard (in other words, $\pi$ is not $\delta$ - simple) 
then for any $\epsilon>0$, no polynomial time algorithm can guarantee a differential approximation ratio greater than, or equal to $\frac{k_{0}}{k_{0}+1}+\epsilon$. We conjecture that $\mathrm{HPP}_{s, t}$ and $\mathrm{HPP}_{s}$ are not $\delta-$ simple and the value $k_{0}$ found for $\mathrm{HPP}_{s, t}$ is smaller that the $k_{0}$ found for $\mathrm{HPP}_{s}$.

Acknowledgments. Many thanks to three anonymous referees for pertinent and useful comments and suggestions.

\section{References}

[1] J. A.Hartigan. Clustering algorithms. New York, Wiley, 1975.

[2] A. Aiello, E. Burattini, M. Massarotti, and F. Ventriglia. A new evaluation function for approximation. Sem. IRIA, 1977.

[3] G. Ausiello, P. Crescenzi, and M. Protasi. Approximate solutions of NP-optimization problems. Theoretical Computer Science, 150:1-55, 1995.

[4] G. Ausiello, A. D'Atri, and M. Protasi. Structure preserving reductions among convex optimization problems. J. of Computer and System Science, 21:136-153, 1980.

[5] C. Bazgan, R. Hassin, and J. Monnot. Differential appoximation for some routing problems (extended abstract). Proc. CIAC 03, LNCS 2653, pages 277-288, 2003.

[6] N. Christofides. Worst-case analysis of a new heuristic for the traveling salesman problem. Technical report 338, Grad. School of Industrial Administration, CMU, 1976.

[7] G. Cornuejols, M. L. Fisher, and G. L. Nemhauser. Location of bank accounts to optimize float: An analytic study of exact and approximate algorithms. Management Science, 23:789-810, 1977.

[8] P. Crescenzi and V. Kann. A compendium of NP-optimization problems. Available on www address: http://www.nada.kth.se/ viggo/problemlist/compendium.html, 2000.

[9] P. Crescenzi and A. Panconesi. Completeness in approximation classes. Information and Computation, 93:241-262, 1991.

[10] M. Demange, D. De Werra, J. Monnot, and V. Th. Paschos. Weighted node coloring: when stable sets are expensive (extended abstract). Proc. WG 02, LNCS 2573, pages 114-125, 2002 .

[11] M. Demange, P. Grisoni, and V. Th. Paschos. Differential approximation algorithms for some combinatorial optimization problems. Theoretical Computer Science, 209:107-122, 1998.

[12] M. Demange, J. Monnot, and V. Th. Paschos. Bridging gap between standard and differential polynomial approxiamtion: the case of bin-packing. Applied Mathematics Letters, 12:127-133, 1999 . 
[13] M. Demange and V. Th. Paschos. On an approximation measure founded on the links between optimization and polynomial approximation theory. Theoretical Computer Science, 158:117-141, 1996.

[14] L. Engebretsen and M. Karpinski. Approximation hardness of TSP with bounded metrics. Manuscript available on www address: http://www.nada.kth.se/ enge/enge-bib/, 2002.

[15] M. L. Fisher, G. L. Nemhauser, and L. A. Wolsey. An analysis of approximations for finding a maximum weight hamiltonian circuit. Operations Research, 27(4):799-809, 1979.

[16] M. R. Garey and D. S. Johnson. Computers and intractability. A guide to the theory of NP-completeness. CA. Freeman, 1979.

[17] R. S. Garfinkel. Motivation and modeling. E. L Lawler, J. K. Lenstra, A. H. G. Rinnooy Kan and D. B. Shmoys (EDs.) The Traveling Salesman Problem: a guided tour of Combinatorial Optimization Wiley Chichester, pages 17-36, 1985.

[18] N. Guttmann-Beck, R. Hassin, S. Khuller, and B. Raghavachari. Approximation algorithms with bounded performance guarantees for the clustered traveling salesman problem. Algorithmica, 28(4):422-437, 2000.

[19] M. Haimovich and A. H. G. Rinnooy Kan. Bounds and heuristics for capacitated routing problems. Mathematics of Operations Research, 10(5):527-542, 1985.

[20] R. HAssin and S. KhulleR. z-approximations. J. of Algorithm, 41(2):429-442, 2001.

[21] R. Hassin and S. Rubinstein. Better approximation for max TSP. Information Processing Letters, 75, 2000.

[22] J. A. Hoogeveen. Analysis of Christofides' heuristic: Some paths are more difficult than cycles. Operations Research Letters, 10:291-295, 1991.

[23] D. S. Johnson and C. H. Papadimitriou. Performance guarantees for heuristics. E. L Lawler, J. K. Lenstra, A. H. G. Rinnooy Kan and D. B. Shmoys (eds) The Traveling Salesman Problem: a guided tour of Combinatorial Optimization WiLEy CHICHESTER 145-180, 1985.

[24] R. M. KarP. Reducibility among combinatorial problems. R. E Miller and J. W. Thatcher, Complexity of Computer Computations, 85-103, Plenum Press, NY, 1972.

[25] R. Kosaraju, J. Park, and C. Stein. tours and short superstrings. Proc. FOCS, 11(4):166$177,1994$.

[26] E. L. Lawler, J. K. Lenstra, A. H. G. Rinooy Kan, and D. B. Shmoys (eds.). The traveling salesman problem: A guided tour of combinatorial optimization. Wiley, New York, 1985. 
[27] J. Monnot. Differential approximation of NP-hard problems with constant size feasible solutions. RAIRO/Operations Research, 36(4):279-297, 2002.

[28] J. Monnot. The maximum $f$-depth spanning tree problem. Information Processing Letters, 80:179-187, 2001.

[29] J. Monnot. Approximation results toward Nearest Neighbor heuristic. Yugoslav Journal of Operations Research, 12(1):11-16, 2002.

[30] P. Orponen and H. Mannila. On approximation preserving reductions: complete problems and robust measures. Technical report, Department of Computer Science, University of Helsinki, 1987.

[31] C. H Papadimitriou and M. Yannakakis. The traveling salesman problem with distance one and two. Mathematics of Operations Research, 18:1-11, 1993.

[32] A. Paz and S. Moran. Non deterministic polynomial optimisation problems and their approximation. Theoretical Computer Science, 95:251-277, 1981.

[33] S. Sahni and T. Gonzalez. P-complete approximation problems. J. of ACM, 23:555-565, 1976.

[34] J. TARhio and E. UkKonen. A greedy approximation algorithm for constructing shortest common superstrings. Theoretical Computer Science, 57:131-145, 1988.

[35] S. A. VAVAsis. Approximation algorithms for indefinite quadratic programming. Mathematical Programming, 57:279-311, 1972.

[36] E. Zemel. Measuring the quality of approximate solutions to zero-one programming problems. Mathematics of Operations Research, 6:319-332, 1981. 